\title{
ALMOST CHEBYSHEV SUBSPACES, LOWER SEMICONTINUITY, AND HAHN-BANACH EXTENSIONS ${ }^{1}$
}

\author{
EDWARD ROZEMA
}

\begin{abstract}
A subset $M$ of a Banach space is called almost Chebyshev iff the set of elements with more than one best approximation from $M$ is the first category. It is first shown that if the metric projection onto a proximinal almost Chebyshev subset $M$ is lower semicontinuous, then $M$ is Chebyshev. Next, let $M$ be a subspace of a separable Banach space. Then $M \perp$ is almost Chebyshev iff the set of elements in $M^{*}$ which fail to have a unique HahnBanach extension is the first category.
\end{abstract}

1. Let $X$ be a Banach space with norm $\|\cdot\|$ and let $M$ be a nonempty subset of $X$. For $x \in X$, we say that $y \in M$ is a best approximation to $x$ from $M$ if

$$
\|x-y\|=\inf \{\|x-m\| ; m \in M\} .
$$

We are interested in examining the set of points in $X$ which have a unique best approximation from $M$. If this set is all of $X$, then $M$ is called Chebyshev.

It is well known that in certain common Banach spaces, for example, $C([0,1] \times[0,1])$ or $L^{1}[0,1]$, there is a dearth of finite dimensional Chebyshev subspaces (none in the second case, only the space of constant functions in the first). A. L. Garkavi [5] inquired into whether, for a given subspace, this nonuniqueness was a rare phenomenon and defined an almost Chebyshev subset to be one for which the set of elements with more than one best approximation is of the first category. S. B. Stechkin [9] had previously shown that any closed nonempty subset of a uniformly rotund Banach space is almost Chebyshev. For a separable Banach space, Garkavi showed that there exist almost Chebyshev subspaces of any finite dimension.

Received by the editors August 7, 1972.

AMS (MOS) subject classifications (1970). Primary 41A50, 41A65; Secondary 46B99.

Key words and phrases. Almost Chebyshev sets, lower semicontinuity, Hahn-Banach extensions, selections, Brown property (P).

${ }^{1}$ This represents work done toward fulfillment of the requirements of the Ph.D. degree of Purdue University, was written under the guidance of Professor R. B. Holmes, and supported by a David Ross University Fellowship.

(c) American Mathematical Society 1973 
In [6], he obtained a characterization of the finite dimensional almost Chebyshev subspaces of $C(T)$ (where $T$ is a compact Hausdorff space) somewhat reminiscent of the classical Haar condition for Chebyshev subspaces. In another paper, we will characterize the almost Chebyshev subspaces of $L^{1}(\mu)$.

This concept is closely allied with another problem of approximation theory concerning the continuity of best approximation. Denote the set of all best approximations to $x$ from $M$ by $P_{M}(x)$ and call the set-valued map $x \rightarrow P_{M}(x)$ the metric projection of $X$ onto $M$. The map $P_{M}$ is lower semicontinuous (1.s.c.) iff, for every open subset $U$ of $X$,

$$
\left\{x \in X ; P_{M}(x) \cap U \neq \varnothing\right\}
$$

is also open. A continuous selection for $P_{M}$ is a continuous map $s: X \rightarrow M$ such that $s(x) \in P_{M}(x)$ for all $x \in X$. E. Michael [7] has shown that if $M$ is convex and $P_{M}$ is l.s.c., then there exists a continuous selection for $P_{M}$. If $M$ is almost Chebyshev then there exists at most one continuous selection for $P_{M}$ since any selection is determined on a dense set. (In this connection it should be pointed out that a recent result of A. L. Brown [4, Theorem 2.8(iii)] giving a sufficient condition for finite dimensional subspaces of $C(T)$ to have at most one continuous selection can be thus derived from Garkavi's characterization of finite dimensional almost Chebyshev subspaces of $C(T)$.) However, Michael's theorem is of little use in proving the existence of a continuous selection for almost Chebyshev subspaces, as the following result shows. (Recall that $M$ is called proximinal if $P_{M}(x) \neq$ $\varnothing$ for each $x \in X$.)

THEOREM 1. Let $M$ be a proximinal subset of $X$. If $P_{M}$ is l.s.c. and $M$ is almost Chebyshev, then $M$ is Chebyshev.

Proof. Suppose $M$ is not Chebyshev. Then there exists an element $x_{0}$ which has more than one best approximation. Let $U_{1}$ and $U_{2}$ be two disjoint open subsets of $X$ for which $P_{M}\left(x_{0}\right) \cap U_{i} \neq \varnothing, i=1,2$. Then, since $P_{M}$ is l.s.c.,

$$
A_{i} \equiv\left\{x \in X ; P_{M}(x) \cap U_{i} \neq \varnothing\right\}
$$

is nonempty and open for $i=1,2$. Thus $A_{1} \cap A_{2}$ is a nonempty open set each element of which has a best approximation in $U_{1}$ and another (necessarily different) best approximation in $U_{2}$. Thus $M$ is not almost Chebyshev.

A. L. Brown has studied 1.s.c. metric projections and introduced the following definition [3]: $X$ has property (P) if whenever $x$ and $z$ are points of $X$ such that $\|x+z\| \leqq\|x\|$, then there exist positive constants $b$ and $c$ such that $\|y+c z\| \leqq\|y\|$ whenever $\|x-y\| \leqq b$. Brown stated that $X$ has property (P) iff $P_{M}$ is l.s.c. for every finite dimensional subspace $M$ of $X$. 
(Recently, the class of subsets $M$ has been enlarged to include the "approximatively-compact and -convex" subsets, see [2].) Brown showed that a rotund space and a finite dimensional space whose unit ball is a finite intersection of half-spaces both have property (P); J. Blatter [1] has shown that $c_{0}$ has property (P). Combining these results with Theorem 1 we obtain the following corollary.

COROLLARY. The only finite dimensional almost Chebyshev subspaces of $c_{0}$ or $l^{1}(\{1, \cdots, n\})$ are Chebyshev.

2. Let $M$ be a subspace of a Banach space $X$ and let $m^{*}$ be a nonzero element of $M^{*}$. We can ask whether $m^{*}$ has a unique norm-preserving extension (called a Hahn-Banach extension) to all of $X$ (in which case we say that $M$ is $\left.\left(U_{m^{*}}\right)\right)$. R. R. Phelps [8] was the first to connect this problem with that of unique best approximation. If, for $m^{*} \in M^{*}$, we set

$$
\operatorname{HBE}_{M}\left(m^{*}\right)=\left\{\text { Hahn-Banach extensions of } m^{*}\right\},
$$

then Phelps, in essence, proved the following result (where $x^{*} \mid M$ is the restriction of $x^{*}$ to $M$ ).

Lemma 1. For all $x^{*} \in X^{*}$,

$$
P_{M}^{\perp}\left(x^{*}\right)=x^{*}-\operatorname{HBE}_{M}\left(x^{*} \mid M\right) .
$$

Following Phelps, we say that $M$ is $(U)$ if $M$ is $\left(U_{m^{*}}\right)$ for every $m^{*} \in M^{*}$. Using the lemma above Phelps was able to conclude the following.

TheORem (Phelps). $M$ is $(U)$ iff $M^{\perp}$ is Chebyshev.

We introduce the following definition: $M$ is almost $(U)$ iff the set of elements in $M^{*}$ which have a unique Hahn-Banach extension is dense and of the second category. We present an analogue to Phelps' theorem in the next result.

THEOREM 2. If $X$ is a separable Banach space, then $M$ is almost $(U)$ iff $M^{\perp}$ is almost Chebyshev.

Proof. Set $L=M^{\perp}$. First, some notation: Let $L_{1}$ be the set of elements in $X^{*}$ which fail to have a unique best approximation out of $L$. Since $X$ is separable, there exists a sequence $\left\{x_{s}\right\}$ of elements in $X$ which separate the elements of $L$. Put

$$
L_{n s}=\left\{x^{*} \in X^{*} ; \sup _{m, m^{\prime} \in P_{L}\left(x^{*}\right)}\left|\left\langle x_{s}, m-m^{\prime}\right\rangle\right| \geqq \frac{1}{n}\right\} .
$$

Since $L$ is proximinal (because it is $w^{*}$-closed), $L_{1}=\bigcup L_{n s}$. By using the facts that the unit ball of $X^{*}$ is $w^{*}$-compact and that the norm is $w^{*}$-lower 
semicontinuous, it can readily be shown that $L_{n s}$ is closed. It is also an easy matter to show that $L_{n s}=L_{n s}+L$ (this only requires the observation that, for $\left.m \in L, P_{L}\left(x^{*}+m\right)=P_{L}\left(x^{*}\right)+m\right)$.

We now suppose that $L=M^{\perp}$ is almost Chebyshev. Thus each $L_{n s}$, being closed, is nowhere dense. Define $R: X^{*} \rightarrow M^{*}$ by $R\left(x^{*}\right)=x^{*} \mid M$. Then $\operatorname{ker} R=L$ and $R$ is an open, continuous map. Lemma 1 shows that

Hence

$$
R\left(L_{1}\right)=\left\{f \in M^{*} ; M \text { is not }\left(U_{f}\right)\right\} .
$$

$$
\left\{f \in M^{*} ; M \text { is not }\left(U_{f}\right)\right\}=R\left(\bigcup L_{n s}\right)=\bigcup R\left(L_{n s}\right) .
$$

We now show that each $R\left(L_{n s}\right)$ is closed and nowhere dense. Since $X^{*} \backslash L_{n s} \equiv L_{n s}^{c}$ is open and dense, it follows that $R\left(L_{n s}^{c}\right)$ is open and dense. So if we can show that $\left(R\left(L_{n s}^{c}\right)\right)^{c}=R\left(L_{n s}\right)$, we will know that $M$ is almost $(U)$. Since $R$ is onto, clearly $\left(R\left(L_{n s}^{c}\right)\right)^{c} \subset R\left(L_{n s}\right)$. To show that opposite inclusion, suppose, on the contrary, that $y \in R\left(L_{n s}\right)$ and $y \in R\left(L_{n s}^{c}\right)$. Choose $x \in L_{n s}$ and $z \in L_{n s}^{c}$ for which $R x=R z=y$. Then there is an $m \in L$ for which $z=x+m$. Since $x \in L_{n s}$ and $m \in L$, it follows from the remarks above that $z \in L_{n s}$, a contradiction.

To prove the other half of the theorem, assume that $M$ is almost $(U)$. Then $A=\left\{f \in M^{*} ; M\right.$ is $\left.\left(U_{f}\right)\right\}$ is dense. Let $R: X^{*} \rightarrow M^{*}$ be the restriction map as above. Then $R^{-1}(A)$ is the set of all $x^{*} \in X^{*}$ which have a unique best approximation from $M^{\perp}$. We claim that $R^{-1}(A)$ is dense: For let $U$ be an open subset of $X^{*}$. Then $R(U)$ is open in $M^{*}$. Thus $R(U) \cap A \neq \varnothing$. Hence $U \cap R^{-1}(A) \neq \varnothing$. Using the remarks and notation above, we have seen that $L_{n s}$ is closed; we have, therefore, just shown that $L_{n s}$ is nowhere dense. Consequently, $L$ is almost Chebyshev.

\section{BIBLIOGRAPHY}

1. J. Blatter, Zur Stetigkeit von mengenwertigen metrischen Projektion, Forshungsber. Landes Nordrhein-Westfalen Nr. 1870, Westdeutscher Verlag, Cologne, 1967, pp. 1738. MR 36 \#3033.

2. B. Brosowski et al., Stetigkeitssätz für metrischen Projektion. II. (P)-Räume und $\mathscr{T}_{\mathrm{s}}$-Stetigkeit der metrische Projektion, Max-Planck-Institut für Physik and Astrophysik 19, München, 1969.

3. A. L. Brown, Best n-dimensional approximation to sets of functions, Proc. London Math. Soc. (3) 14 (1964), 577-594. MR 29 \#5033.

4. - On continuous selections for metric projections in space of continuous functions, J. Functional Analysis 8 (1971), 431-449.

5. A. L. Garkavi, On Čebyšev and almost-Čebyšev spaces, Izv. Akad. Nauk SSSR Ser. Mat. 28 (1964), 799-818; English transl., Amer. Math. Soc. Transl. (2) 96 (1970), 153175. MR 29 \#2635; MR 42 \#2900.

6. - Almost-Čebyšev systems of continuous functions, Izv. Vysš. Učebn. Zaved. Matematika 1965, no. 2 (45), 36-44; English transl., Amer. Math. Soc. Transl. (2) 96 (1970), 177-187. MR 32 \#1550. 
7. E. Michael, Continuous selections. I, Ann. of Math. (2) 63 (1956), 361-382. MR 17, 990.

8. R. R. Phelps, Uniqueness of Hahn-Banach extensions and unique best approximation, Trans. Amer. Math. Soc. 95 (1960), 238-255. MR 22 \#3964.

9. S. B. Stečkin, Approximative properties of sets in normed linear spaces, Rev. Math. Pures Appl. 8 (1963), 5-18. (Russian) MR 27 \#5108.

Department of Mathematics, Purdue University, Calument Campus, Hammond, INDIANA 46323 